\title{
Compensating Losses in Doped Negative-Index Metamaterials via Four-Wave Mixing and Quantum Control
}

\author{
Alexander K. Popov ${ }^{\dagger}$, Sergey A. Myslivets*, Thomas F. George ${ }^{\star}$, and Vladimir M. Shalaev \\ ${ }^{\dagger}$ University of Wisconsin-Stevens Point, Stevens Point, WI 54481; 'Institute of Physics of Russian Academy of Sciences, 660036 Krasnoyarsk, \\ Russia; "University of Missouri-St. Louis, St. Louis, MO 63121; ${ }^{\ddagger}$ Purdue University, West Lafayette, IN 47907 \\ apopov@uwsp.edu Tel: +1(920)969-0778
}

\begin{abstract}
All-optical switching from absorption to amplification and wavelength-selective narrowband filtering are shown possible in doped solids based on parametric amplification and quantum interference. The results are discussed in the context of compensation of losses in negative-index metamaterials.

(C)2007 Optical Society of America

OCIS codes: (270.1670) Coherent optical effects, (1904410) Nonlinear optics, parametric processes
\end{abstract}

Absorption is generally recognized now as one of the most challenging problems that needs to be addressed for practical applications of negative-index metamaterials (NIMs) - artificial electromagnetic materials that promise revolutionary breakthrough in photonics. One of the possible approaches is compensating losses by the amplifying centers embedded into a NIM host material to provide amplification due to population inversion [1]. An alternative approach based on optical parametric amplification in NIMs has been proposed in [2], where the precondition is the non-symmetric current-voltage characteristics of NIM's structural elements that give rise to its quadratic nonlinearity.

Here, we investigate a different option that does not require strong nonlinear-optical response by the building blocks of the NIM. Instead, it employs strong resonant nonlinearities inherent to embedded four-level centers (like molecules and ions) and the methods of quantum control attributed to four-wave coupling in resonant schemes. The coupling scheme under investigation (Fig. 1) involves interference between resonant Raman-like and optical parametric amplification-like quantum pathways [3] (and references therein), but does not rely on a coherent population trapping-type of excitation commonly employed in double- $\Lambda$ schemes of quantum control in solids [4]. The options of spin (ions) and vibration coherence (molecules) $l n$ are analyzed and compared.

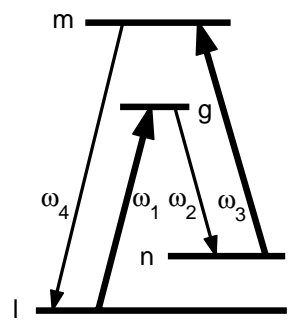

Fig. 1. Amplification at $\omega_{4}$ controlled by two lasers at $\omega_{1}$ and $\omega_{3}$ Idler at $\omega_{2}$ may experience strong Raman amplification.

We also show that the propagation properties of the signal (probe) wave become dramatically different in NIM compared with its counterpart in natural crystals. Counterintuitive features of nonlinear-optical processes under investigations originate from opposite directions of the energy flow and the phase velocity and from corresponding effects similar to distributed feedback, which is inherent to electromagnetic waves propagating in NIMs. The feasibilities of compensation of strong losses in negative- and positive-index materials by two laser beams as well as of wavelength-selective frequency-tunable narrow-band filtering are shown and illustrated through numerical experiments.

\section{References}

[1] V. M. Shalaev, "Optical negative-index metamaterials," Nature Photonics, 1, 41-48 (2007).

[2] A. K. Popov, S. A. Myslivets, and T. F. George, "Nonlinear interference effects and all-optical switching in optically dense inhomogeneously broadened media," Phys. Rev. A 71, 043811-1-13 (2005).

[3] A. K. Popov and V. M. Shalaev, "Compensating losses in negative-index metamaterials by optical parametric amplification," Opt. Lett. 31, 2169-2171 (2006); http://arxiv.org/abs/physics/0601055.

[4] B. S. Ham and P. R. Hemmer, "Coherence switching in a four-level system: Quantum switching," Phys. Rev. Lett. 84, 4080-4083 (2000). 